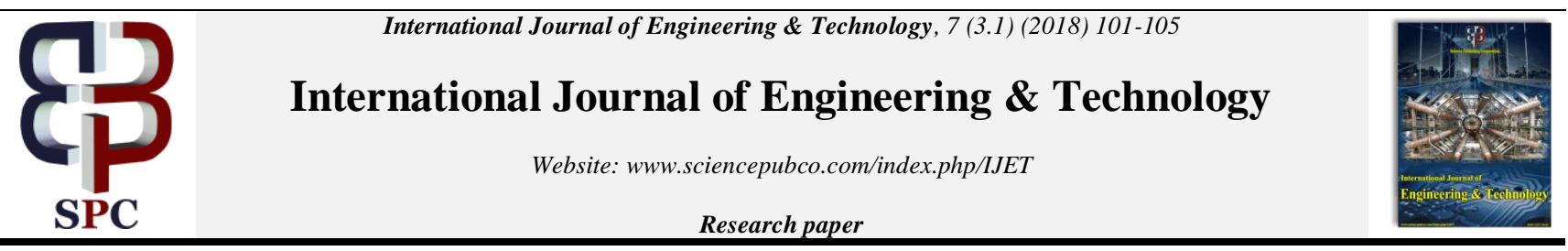

\title{
Design, Implementation and Analysis of 8T SRAM Cell in Memory Array
}

\author{
B. Kaleeswari ${ }^{1}$, S. Kaja Mohideen ${ }^{2}$ \\ ${ }^{1,2}$ Department of ECE, B.S. Abdur Rahman University, Chennai, Tamilnadu-600048, India. \\ *Corresponding author E-mail: kaleeswariece@gmail.com
}

\begin{abstract}
In modern VLSI designs, static random access memory plays a vital role because of its high performance and low power consumption qualities. As technology is scale down, the importance of the power analysis and leakage current of memory design is increasing. This paper describes about the $1 \mathrm{~KB}$ size memory design using SRAM. The proposed design of 8T SRAM single cell in implemented in array structure of size $32 \times 32$.The design structure reduces the power by $75 \%$ by reducing the leakage current. The proposed $8 \mathrm{~T}$ SRAM cell is implemented and analyzed in $90 \mathrm{~nm}$ technology using Digital schematic and Micro wind software.
\end{abstract}

Keywords: $W L, B L, B L B$ and SRAM

\section{Introduction}

In today's world, the information technology is depending on semiconductor based electronics. However the first transistor was invented in 1948, there will be a tremendous growth in semiconductor industry. There are two major fields which are benefited by the growth of semiconductor industry, are semiconductor based memories and microprocessors. The performance has improved by this technological advancement parallel the device density also increases.

The power dissipation in integrated circuit(IC) depending on different operating modes of the circuit first one is during active mode of operation the dynamic power is dominating. Second one is two primary leakage sources the active and standby leakage component.

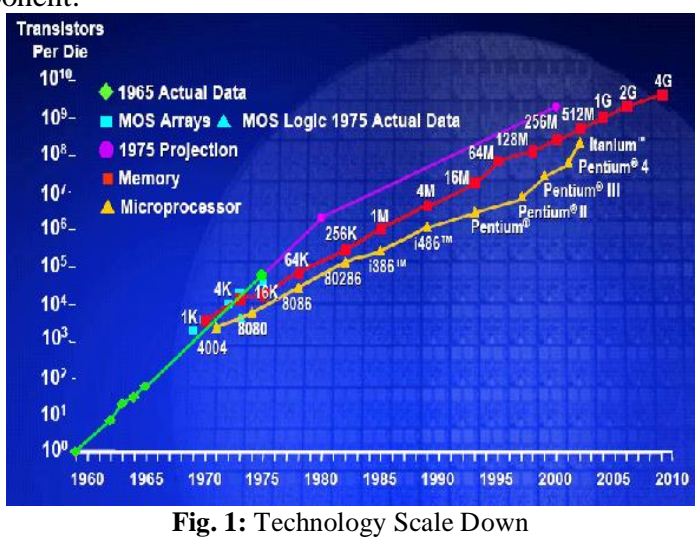

Section 1, describes about the introduction to VLSI circuits. Section 2, explains about the basic operation of SRAM. Section 3 briefs about the effects of power dissipation. Section 4 , discusses about the proposed 8T SRAM circuit. Section 5, describes about the memory array architecture. Section 6 discusses about the im- plementation of $8 \mathrm{~T}$ circuit in array structure. Section 7 , discusses about the simulation and result .finally section 8 concludes the work.

\section{Basics of SRAM}

In static RAM circuit the data can be stored perpetually until the power supply is ON.In static RAM array; single storage cell always consists of simple latch circuit, in which two stable operating points are present. Figure 2 shows the single SRAM cell.

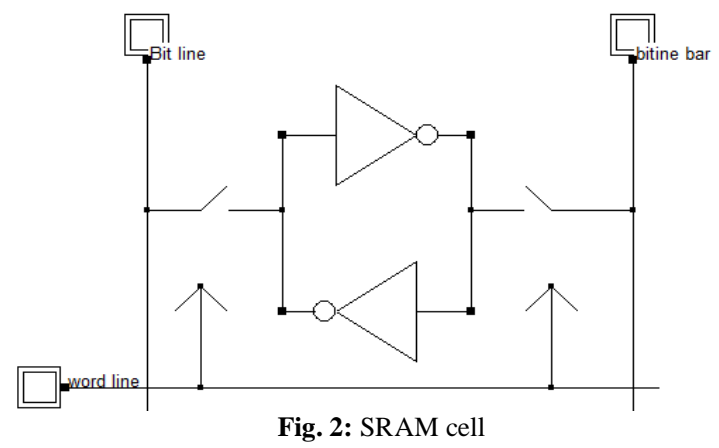

Based on conserved state of two inverter latch circuit, the data can be interpreted as logic ' 1 ' or logic ' 0 'depending upon the data being held on the memory. Data contained in memory can be accessed through the bit line and bit line bar. In order to write a data in a memory a switch is used called word line.

\subsection{CMOS SRAM Cell Design}

The Single SRAM cell using CMOS transistor is shown in Fig 3. To design the low power CMOS inverter, use cross coupled CMOS inverter is used. The static power dissipation is very small in cross coupled inverter circuit. However there is the considerable amount of leakage current in the circuit. A simple CMOS inverter is connected back to back and two access transistors are present 
in a single memory cell. Whenever the word line is activated the two access transistors are turned ON [6].

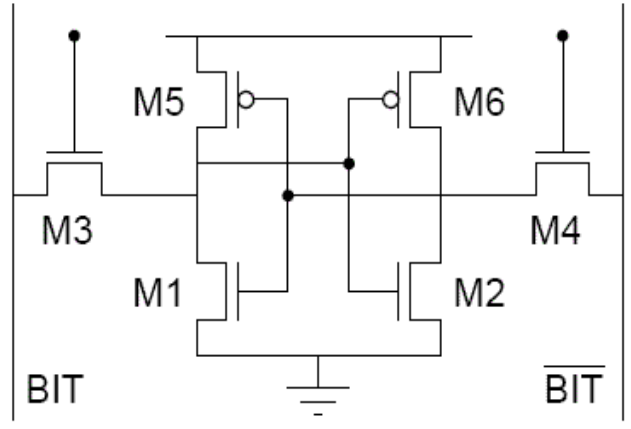

Fig. 3: SRAM Cell using CMOS

\subsection{SRAM Read Operation}

Initially a logic 0 is stored in the cell, the transistor M1 and M6 operates in linear region, and M2 and M5 in cutoff region .This results in the internal node $\mathrm{V}_{1}=0 \mathrm{v}$ and $\mathrm{V}_{2}=\mathrm{V}_{\mathrm{dd}}$, before two access transistor get turn $\mathrm{ON}$.This is shown in fig 4.Using row column circuitry, the two access transistors $\mathrm{M}_{3}$ and $\mathrm{M}_{4}$ are turned $\mathrm{ON}$.

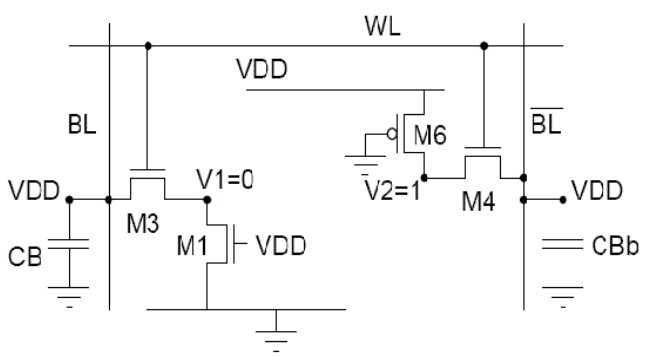

Fig. 4: Read Operation

The voltage at bit line bar will not change since there is no current flow through $\mathrm{M}_{4}$.on the other side the $\mathrm{M}_{1}$ and $\mathrm{M}_{3}$ will conduct a nonzero current .The voltage level at the capacitance of bit line will drop slightly. The voltage at $\mathrm{V}_{1}$ will increase from ' 0 ' $\mathrm{v}$, however the $\mathrm{V}_{1}$ may exceeds the threshold voltage of $\mathrm{M}_{2}$ during this process flipping of states will occur . Therefore $\mathrm{V}_{1}$ must not exceeds the threshold voltage of $\mathrm{M}_{2}$ so the transistor $\mathrm{M}_{2}$ remain turn off during read operation.

$\mathbf{V}_{1 \max } \leq \mathbf{V}_{\mathrm{T}, 2}$

The transistor M3 is in saturation whereas M1 is linear, equating the current equations we get

$$
\frac{\beta_{M N 3}}{2}\left(V_{D D}-V_{1}-V_{T N}\right)^{2}=\frac{\beta_{M 11}}{2}\left(2\left(V_{D D}-V_{T N}\right) V_{1}-V_{1}^{2}\right)
$$

Substitute Eqn1 in Eqn 2

$\frac{\beta_{M N 3}}{\beta_{M 11}}=\frac{(W / L)_{3}}{(W / L)_{1}} \leq 2 V_{I N} \frac{\left(V_{D D}-1.5 V_{T N}\right)}{\left(V_{D D}-2 V_{T N}\right)^{2}}$

\subsection{SRAM Write Operation}

Assume that logic 0 has to written in the cell, consider a logic 1 is stored in the cell .Transistor M2 and M5 are operates in linear region and $\mathrm{m} 1$ and $\mathrm{M} 5$ are operating in cutoff region.Before the two access transistor turn $\mathrm{ON}$, the node voltage at $\mathrm{V}_{1}=\mathrm{V}_{\mathrm{dd}}$ and $\mathrm{V}_{2}=0$. The bitline voltage $\mathrm{V}_{\mathrm{b}}$ is forced to 0 by write column circuitry. After the transistor M3 and M4 turns ON according to the equation $\mathrm{V}_{1 \max }<=\mathrm{V}_{\mathrm{T}, 2}$.

The node voltage at $\mathrm{V}_{2}$ remains below the threshold voltage of $\mathrm{M}_{1}$. The voltage at node 2 would not be sufficient .In order to store logic 1 force $\mathrm{V}_{1}=0$ and $\mathrm{V}_{2}=\mathrm{V}_{\mathrm{dd}}$ this in turn off the transistor $\mathrm{M}_{2}$ due to reduction in voltage $V_{1}$ below the threshold level .When
$\mathrm{V}_{1}=\mathrm{V}_{\mathrm{t}}$, transistor $\mathrm{M}_{3}$ operates in linear region while $\mathrm{M}_{5}$ operates in saturation region

$\frac{\beta_{P 5}}{2}\left(0-V_{D D}-V_{T P}\right)^{2}=\frac{\beta_{M 13}}{2}\left(2\left(V_{D D}-V_{I D}\right) V_{1}-V_{1}^{2}\right)$

Rearranging the condition of $\mathrm{V}_{1}$ in the result we get

$$
\frac{\beta_{P 5}}{\beta_{M 3}}=\frac{\mu_{P}}{\mu_{N W}} \cdot \frac{(W / L)_{5}}{(W / L)_{3}}<\frac{\left(2\left(V_{D D}-1.5 V_{T N}\right) V_{T Z}\right)}{\left(V_{D D}-V_{I P}\right)}
$$

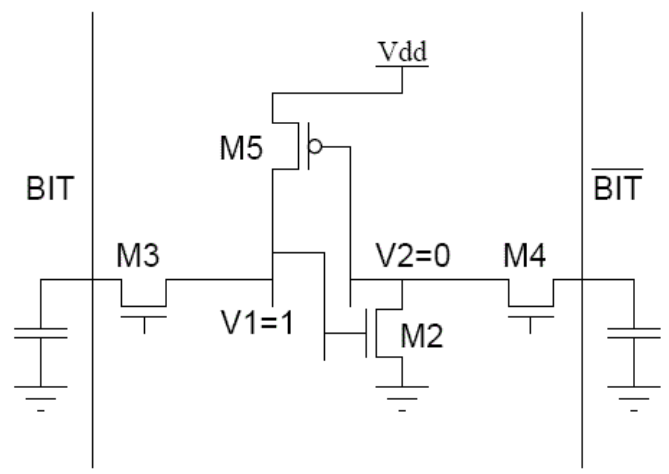

Fig. 5: Write Operation

\section{Effects of Power Dissipation}

The When power is dissipated, it invariably leads to rise in temperature of the chip. This rise in temperature affects the device both when the device is off as well as when the device is on.

When the device is off, it leads to increase in the number of intrinsic carriers, by the following relation:

$\boldsymbol{n}_{i} \propto e^{\frac{-E G}{k T}}$

From the above equation it is observed that whenever there is increase in temperature the number of intrinsic carriers is also increased. The majority carriers are not affected by temperature change because the majority carriers are contributed by impurity atoms. When temperature increases the concentration of minority carriers get increased this in turn leakage current in the device get increased which in turn further increase in temperature.

\subsection{Sources of Leakage Power}

No there are four sources of leakage power in CMOS circuit

(i) Reverse biased junction leakage current $\left(\mathrm{I}_{\mathrm{REV}}\right)$

(ii) Gate induced Drain Leakage $\left(\mathrm{I}_{\mathrm{GIDL}}\right)$

(iii)Gate direct tunneling $\operatorname{effect}\left(\mathrm{I}_{\mathrm{G}}\right)$

(iv)Sub threshold leakage $\left(\mathrm{I}_{\mathrm{SUB}}\right)$

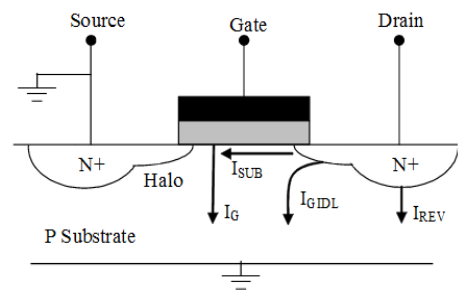

Fig 6: Leakage Current Components

\subsection{Sub Threshold Leakage Current}

In weak inversion region, the drain source current of transistor is operating. The main reason for occurring the sub threshold condition is diffusion current of minority carriers in the channel. In CMOS inverter the logic ' 0 ' input is given to NMOS transistor ,it 
turns to off and output voltage is high. However $\mathrm{V}_{\mathrm{GS}}=0 \mathrm{v}$ thereis current flow in the channel of NMOS transistor due to $\mathrm{V}_{\mathrm{DD}}$. Because the sub threshold voltage depends on supply voltage, Temperature, device size and process parameter. Over which the threshold voltage plays a vital role.

\subsection{Effect of Sub Threshold Leakage Current}

The sub threshold leakage current $\mathrm{I}_{\mathrm{SUB}}$ has a greater contribution for power dissipation than the other leakage current in CMOS technologies.

$\mathrm{I}_{\mathrm{SUB}}$ is calculated using the formula

$$
I_{S U B}=\frac{W}{L} \mu V_{t h}^{2} C_{S t h e} e^{\frac{V_{C S S}-V_{T}+N_{D S}}{N_{t h s}}}\left(1-e^{\frac{-V_{D S}}{V_{t S h}}}\right)
$$

Where $\mathrm{W}$ and $\mathrm{L}$ be the width and length of transistor, $\mu$ denotes the mobility of carriers, Vth is threshold voltage $\eta$ is the drain induced barrier lowering coefficient

$$
n=1+\frac{C_{s t h}}{C_{o x}}
$$

Where $\mathrm{C}_{\mathrm{ox}}$ is gate input capacitance per unit area of MOS gate. When long channel transistor with $\mathrm{V}_{\mathrm{DS}}$ larger than $\mathrm{V}_{\mathrm{th}}$ is in off state

$$
I_{S U B}=\frac{W}{L} \mu v_{t h}^{2} C_{s t h} 10^{\frac{-V_{T}}{S}}
$$

Where $\mathrm{S}$ denotes the sub threshold swing parameter .It is highly desirable that $\mathrm{S}$ should be small as possible because it is going to determine the amount of voltage swing necessary to switch ON and OFF the MOSFET (typical values of S for bulk CMOS devices are $70-110 \mathrm{mV} /$ decade; the theoretical lower bound is 60 $\mathrm{mV} /$ decade corresponding to $\mathrm{n}=1$.)Increase in temperature results in larger $S$ value this in turn increase the OFF.

In long channel devices there is a small amount of current flow due to the influence of $\mathrm{I}_{\mathrm{DS}}$. In short channel devices the channel lengths get reduced results in overlapping the source and drain depletion region get overlapped. The electrons will flow in greater amount with a small amount of charge in gate cause decrease in threshold voltage and increase in $\mathrm{I}_{\mathrm{OFF}}$.

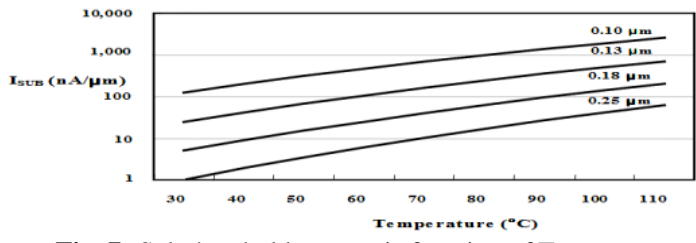

Fig. 7: Sub threshold current is function of Temperature

From the figure it is clearly understand that whenever there is increase in temperature there will be increase in sub threshold leakage current by a factor of 10 and decrease in threshold voltage by $100 \mathrm{mV}$. As a result a device having low threshold voltage due to process variation undergone more leakage current.

\section{Proposed 8T SRAM Circuit}

By considering all the above factors that cause sub threshold leakage current ,the new circuit was proposed as shown if fig 8 . The proposed 8T SRAM circuit consists of single 6T SRAM cell with two PMOS is added with pass transistors.

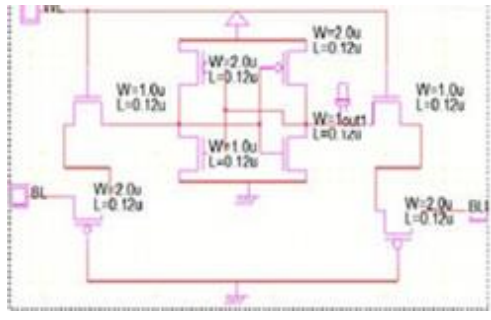

Fig. 8: Proposed 8T SRAM Circui

In the proposed 8T SRAM circuit the sub threshold leakage current is reduced by two PMOS transistor connected the source terminal of two pass transistors.

\subsection{Read Operation}

During read operation the voltage at node $\mathrm{V}_{1}$ (refer to fig 3 ) will not exceeds the threshold voltage of $\mathrm{M} 2$ transistor .But practically V1 voltage will exceeds the threshold voltage of M2 due to sub threshold leakage current caused by shrinking of device geometry. This will results in flipping of states and power dissipation also.

This effect will be avoided by introduce gate delay in the circuit. The gate delay will be introduced by increasing the channel length of two pass transistors .But this will increase the $\mathrm{I}_{\mathrm{oN}}$ current .By considering all this into account two PMOS transistors are introduced. This two PMOS transistor introduce the delay in pass transistors, in order to maintain the $\mathrm{I}_{\mathrm{OFF}}$ less than the threshold voltage of M2 transistor.

\subsection{Write Operation}

During write operation, in order to store logic 1 force $\mathrm{V}_{1}=0$ and $\mathrm{V}_{2}=\mathrm{V}_{\mathrm{dd}}$ (refer to fig 4) this in turn off the transistor $\mathrm{M}_{2}$ due to reduction in voltage $\mathrm{V}_{1}$ below the threshold level. When $\mathrm{V}_{1}=\mathrm{V}_{\mathrm{t}}$, transistor $\mathrm{M}_{3}$ operates in linear region while $\mathrm{M}_{5}$ operates in saturation region. The same condition prevails in read operation as discussed in above paragraph, $\mathrm{I}_{\mathrm{OFF}}$ current will starts increasing this in turn exceeds the threshold voltage of M2 transistor results in flipping of states. This will be avoided by two PMOS transistor connected to pass transistor introduce delay .by introducing delay the $\mathrm{V}_{2}$ is kept below the threshold voltage of M2 in order to reduce the power dissipation.

\subsection{Analysis}

The sub threshold leakage current is defined as

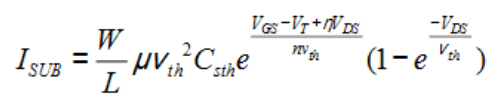

From the equation it is clearly shows that the sub threshold leakage current is reduced by

(i) Decreasing the width of transistor.

(ii) Decreasing the mobility of charge carriers.

(iii) Reducing threshold voltage.

(iv) Exponential function of gate source voltage and drain source voltage.

Without changing the device geometry the sub threshold leakage current is reduced by proposed 8T SRAM circuit. The reason behind the without changing the device geometry is this will turn induce the second order effects of MOS transistors and fabrication of device is also too complicated. 


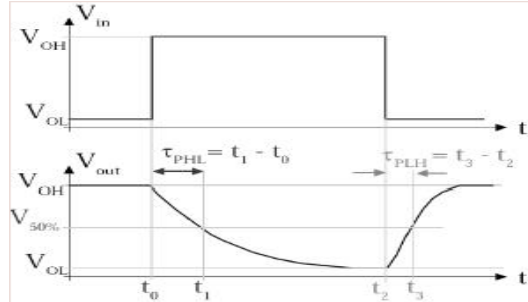

Fig. 9: Propagation Delay Graph

In exponential function of above equation the drain to source voltage $\mathrm{V}_{\mathrm{DS}}$ and gate to source voltage is smaller value than $\mathrm{V}_{T}$ cause exponential reduction in sub threshold leakage current. In the proposed circuit this will be achieved by introduce the delay in the pass transistor circuit this will turn reduce the $\mathrm{V}_{\mathrm{GS}}$ and $\mathrm{V}_{\mathrm{DS}}$ The propagation delay of transistor is calculated by formula

$\tau_{p}=\left(\tau_{p L H}+\tau_{p H L}\right) / 2$

$\tau_{\mathrm{pLH}}$ - Low to high transition

$\tau_{\mathrm{pHL}}-$ High to Low transition

\section{Memory Array Architecture}

The memory arrays are organized such a way that the horizontal and vertical dimensions of same order of magnitude in order to get the aspect ratio equals to one. Figure 10 describes the overview of SRAM memory design. A single address of $\mathrm{N}+\mathrm{M}$ bits is split into $\mathrm{N}$ row address and $\mathrm{m}$ column address. The row address is first decoded followed by column address.

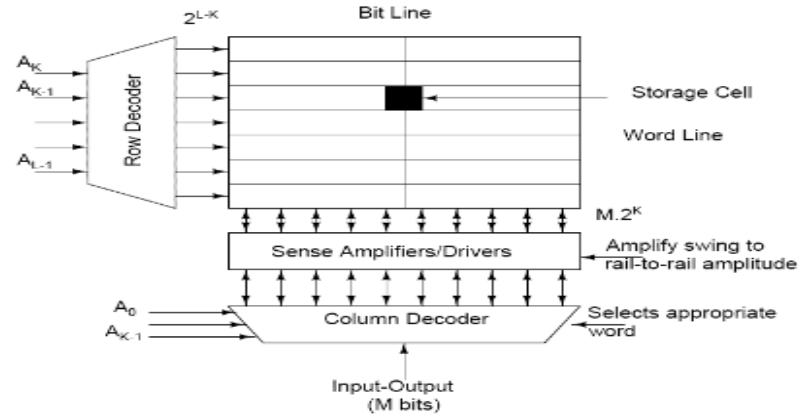

Fig. 10: Memory with Row and Column Decoders

\subsection{Sections of Memory Array}

The Memory Array consists of

1. Pre Charge Circuit

2. Array Structure

3. Row Decoder

4. Column Decoder

5. Sense Amplifier/Driver

1. Pre Charge Circuit: Pre charge circuit is one of important component in SRAM . The function of pre charge circuit is it enables the bit lines to charge to $\mathrm{V}_{\mathrm{dd}}$ except during read and write operation.

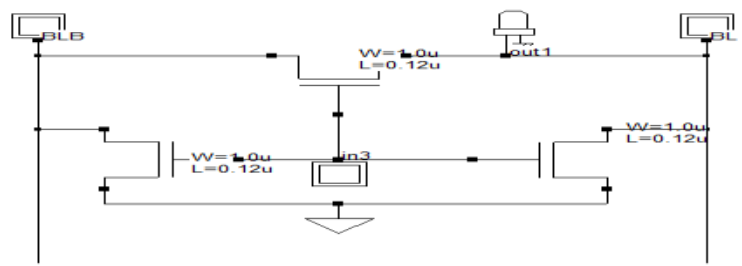

Fig. 11: Pre Charge Circuit

2. Row and Column Decoder: Row decoder is a circuit as shown in figure 12 , which is used to select the particular row in a memory array. Similarly column decoder is used to select the particular column.

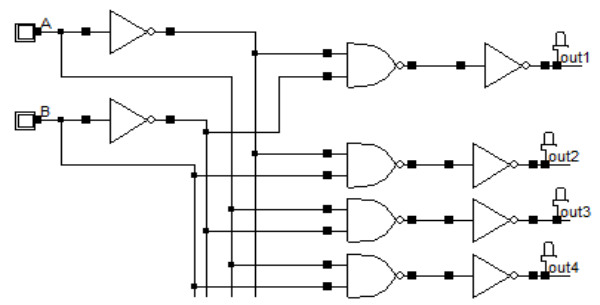

Fig. 12: Row and Column Decoder

3. Sensing Amplifier: The design of sensing amplifier plays a major role while deigning memories. The access time of memory is depends on the performance of sensing amplifier. When $\mathrm{SE}=1$, the amplifier senses the differences between Bit and bit bar and produces the voltage.

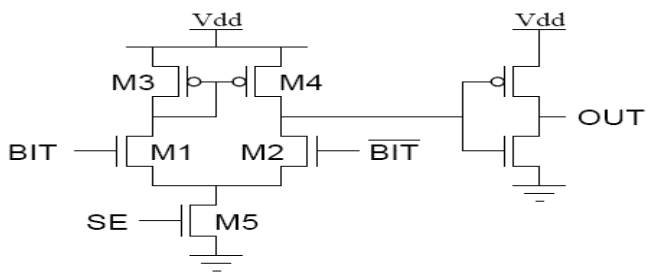

Fig. 13: Sensing Amplifier

\section{Implementation of Proposed Circuit in Array Structure}

The proposed 8T SRAM cell is implemented in array structure in different sizes and parameters are analyzed. Figure shows the $8 \times 8$ array structure analysis using proposed circuit.

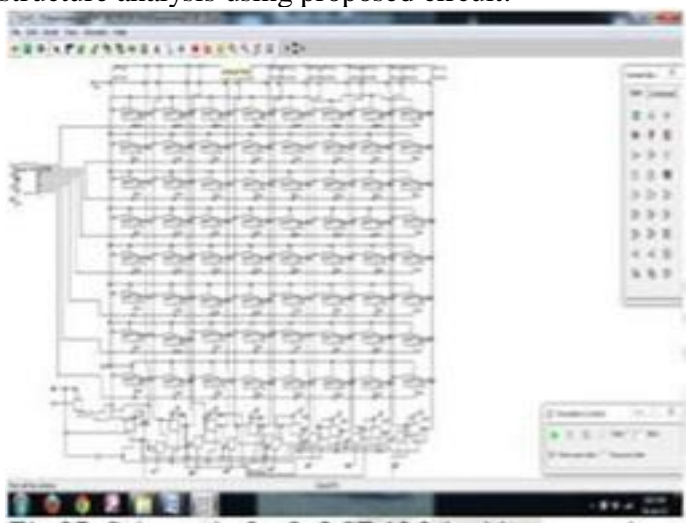

Fig. 14: Array structure using 8T SRAM cell

The memory array is constructed using 3:8 decoder for row and column decoder circuit and latch type differential sensing circuit is used .consider that for writing data in array circuit first select the row address followed by column address then set the data in bit line and bit line bar that is read by sensing amplifier and produce the output voltage.

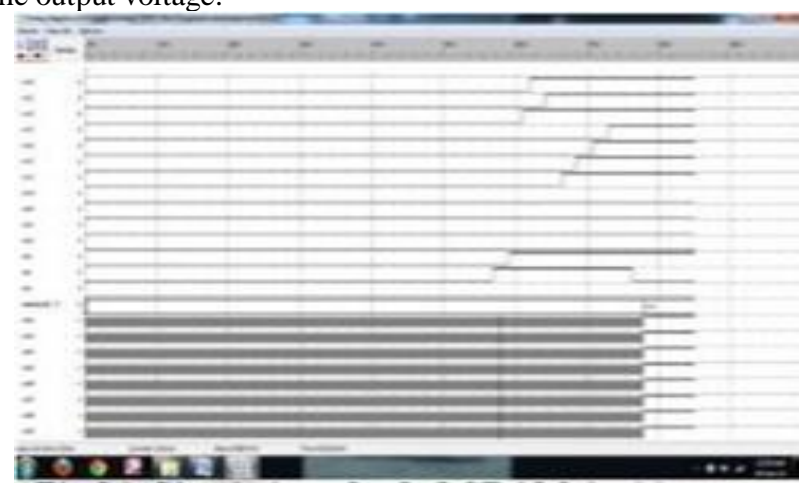

Fig. 15: Timing Analysis of $8 \times 8$ SRAM Array 


\section{Simulation and Results}

By simulating the SRAM cell under different approaches at $\mathrm{Vdd}=1 \mathrm{v}$ and $\mathrm{T}=25^{\circ} \mathrm{c}$ Table 1 show the power comparison of different approaches for reducing the sub threshold leakage current in an SRAM array.

\begin{tabular}{|c|c|c|c|c|c|c|}
\hline Arr & $6 \mathrm{~T}$ & P. & G- & Stacki & $9 \mathrm{~T}$ & Propos \\
\hline ay & SRA & gate(u & gate(u & $\mathrm{ng}$ (uw) & SRA & ed $8 T$ \\
\hline size & $\begin{array}{l}\text { M } \\
\text { (uw) }\end{array}$ & w) & w) & & $\begin{array}{l}\text { M(uw } \\
\text { ) }\end{array}$ & $\begin{array}{l}\text { SRAM } \\
\text { (uw) }\end{array}$ \\
\hline $2 \times 2$ & 495 & 950 & 935 & 224 & 154 & 125 \\
\hline $4 \times 4$ & 666 & 1957 & 1256 & 468 & 234 & 195 \\
\hline $8 \times 8$ & 845 & 3286 & 3134 & 624 & 350 & 258 \\
\hline $\begin{array}{l}16 \mathrm{x} \\
16\end{array}$ & 1026 & 5342 & 5256 & 842 & 438 & 432 \\
\hline $\begin{array}{l}32 \mathrm{x} \\
32\end{array}$ & 1253 & 8436 & 5436 & 1024 & 958 & 512 \\
\hline
\end{tabular}

The graphical representation of Table 1 is shown in figure 16.power dissipation is compared between 6T, stacking technique, $9 \mathrm{t}$ and proposed 8T SRAM structure in an array.

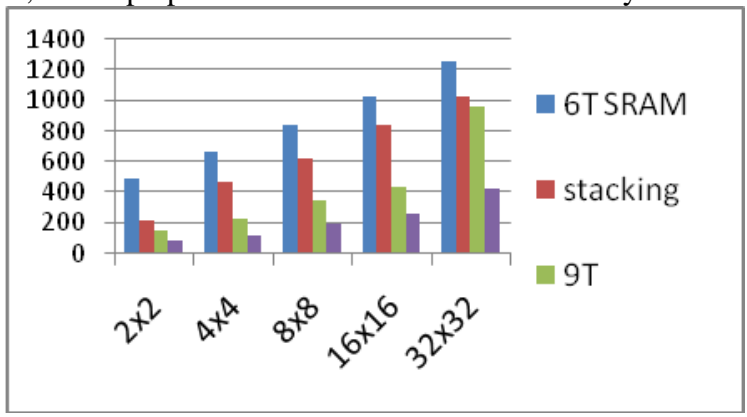

Fig. 16: Comparison of Power

Table 2 show the delay calculation of different approaches for reducing the sub threshold leakage current in an SRAM array.

Table 2: Comparison of Delay in SRAM Array

\begin{tabular}{|c|c|c|c|c|c|c|}
\hline $\begin{array}{l}\text { Array } \\
\text { size }\end{array}$ & $\begin{array}{l}6 \mathrm{~T} \\
\text { SRA } \\
\text { M } \\
\left(\mathrm{um}^{2}\right)\end{array}$ & $\begin{array}{l}\text { P- } \\
\text { gate } \\
\left(u^{2}\right)\end{array}$ & $\begin{array}{l}\text { G- } \\
\text { gate } \\
\left(u^{2}\right)\end{array}$ & $\begin{array}{l}\text { Stackin } \\
\mathrm{g} \\
\left(\mathrm{um}^{2}\right)\end{array}$ & $\begin{array}{l}\text { 9T } \\
\text { SRA } \\
\text { M } \\
\left(\mathrm{um}^{2}\right)\end{array}$ & $\begin{array}{l}\text { Propo } \\
\text { sed 8T } \\
\text { SRA } \\
\text { M(um } \\
\text { 2) }\end{array}$ \\
\hline $2 \times 2$ & 73 & 78 & 83 & 653 & 92 & 84 \\
\hline $4 \times 4$ & 288 & 310 & 314 & 16784 & 464 & 325 \\
\hline $8 \times 8$ & 1148 & 1160 & 1154 & 64324 & 1468 & 1279 \\
\hline $\begin{array}{l}16 \times 1 \\
6\end{array}$ & 4645 & 4828 & 1245 & 248436 & 5942 & 5210 \\
\hline $\begin{array}{l}32 \times 3 \\
2\end{array}$ & 18464 & $\begin{array}{l}1924 \\
8\end{array}$ & $\begin{array}{l}1952 \\
6\end{array}$ & $\begin{array}{l}356324 \\
1\end{array}$ & 24326 & 20840 \\
\hline
\end{tabular}

From the Table it clearly shows that the delay values get reduced in considerable amount while comparing with other technique. Table 3 show the area calculation of different approaches for reducing the sub threshold leakage current in an SRAM array.

From the Table 3 it clearly shows that the area values get slightly increased in considerable amount while comparing with 9T and $6 \mathrm{~T}$ technique but less than stacking technique.

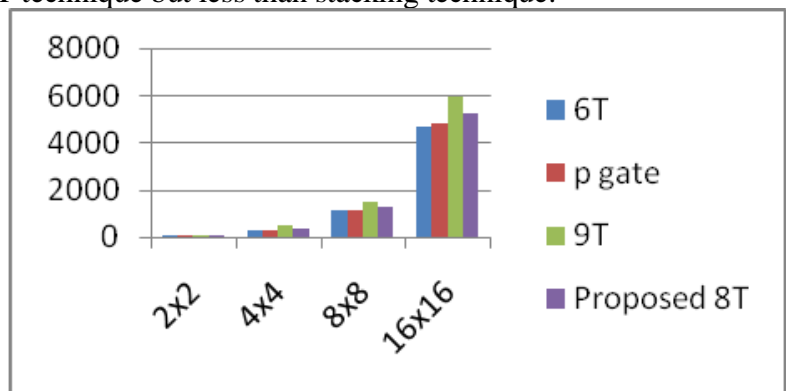

Fig. 17: Comparison of Area
Table 3: Comparison of Area

\begin{tabular}{|c|c|c|c|c|c|c|}
\hline Arr & $6 \mathrm{~T}$ & P. & G- & Stacki & 9T & Proposed \\
\hline ay & SRAM & gate & gate & $\mathrm{ng}$ & SRAM & $8 \mathrm{~T}$ \\
\hline size & & & & & & SRAM \\
\hline $2 \times 2$ & $382 \mathrm{~ns}$ & $364 \mathrm{~ns}$ & $395 \mathrm{~ns}$ & $412 \mathrm{~ns}$ & $376 \mathrm{~ns}$ & $344 \mathrm{~ns}$ \\
\hline $4 x 4$ & $763 \mathrm{~ns}$ & $725 \mathrm{~ns}$ & $784 \mathrm{~ns}$ & $826 \mathrm{~ns}$ & $756 \mathrm{~ns}$ & $688 \mathrm{~ns}$ \\
\hline $8 \mathrm{x} 8$ & $1232 \mathrm{~ns}$ & 1195 & $1295 \mathrm{~ns}$ & $1520 \mathrm{~ns}$ & $1426 \mathrm{~ns}$ & $1081 n s$ \\
\hline & & ns & & & & \\
\hline $16 \mathrm{x}$ & 82us & 76us & 89 us & 115 us & 82us & 64us \\
\hline 16 & & & & & & \\
\hline $32 x$ & 240us & 220 us & $222 \mathrm{us}$ & 274us & 212us & 186us \\
\hline 32 & & & & & & \\
\hline
\end{tabular}

\section{Conclusion}

The leakage current contributes $40 \%$ of total power dissipation in a memory cell. Proposed technique will reduce the power dissipation by $75 \%$ as compared to other technique .By reducing the sub threshold current has greater impact on power dissipation. This technique will reduce the delay by considerable amount and slight increase in area which is also acceptable amount only Simulation carried out in $90 \mathrm{~nm}$ technology that is well suitable for industrial applications.

\section{References}

[1] Tae Woo Oh, Han wool Jeong, Kyoman Kang, Juhyun Park, Younghwi Yang, and Seong-Ook Jung, Power-Gated 9T SRAM Cell for Low-Energy Operation" IEEE Transactions On Very Large Scale Integration Systems (2016).

[2] Zhou Keji, Wang Pengjun "Design of power balance SRAM for DPA-resistance" Journal of Semiconductors, Vol.37, No.4, April 2016.

[3] B. Kaleeswari, Dr. S. KajaMohideen ,"Analysis of Leakage current in 8T SRAM for low power application "Journal of social, technological and environmental sciences, Sep 2017,Vol No 6 pp-601-610.

[4] Jaspreet Kaur, Candy Goyal," Comparative Analysis of Low Leakage SRAM Cell at 32nm Technology" International Journal of Computer Applications, Volume 133 - No.12, January 2016.

[5] Shalini Singh, Vishwas Mishra, Low Power Consuming $1 \mathrm{~KB}$ (32x32) Memory Array Using Compact 7T SRAM Cell, International Journal of Advanced Engineering and Global Technology Vol-04, Issue-01, January 2016.

[6] G. Apostolidis, D. Balobas and N. Konofaos, "Design and Simulation of 6T SRAM Cell Architectures in 32nm Technology" Journal of Engineering Science and Technology Review Jan 2016, pp-145 149.

[7] AbhishekMathur, ArunJayachandran, RamyaVenumbaka "Low Leakage SRAM design using sleep transistor stack”, (2013).

[8] P.S.G. SRIDEVI, P.V.K. CHAITANYA," New Leakage Reduction Techniques", International Journal of Advances in Science Engineering and Technology, Volume- 1, Issue- 1, July-2013.

[9] Saurabh Khandelwal, Balwinder Raj Leakage Current and Dynamic Power Analysis Of Finfet Based 7T SRAM At 45nm Technology, The International Arab Conference on Information Technology (ACIT'2013).

[10] Atluri .Jhansi rani, K .Harikishore, Fazal Noor Basha,,V.G. SanthiSwaroop, L. VeeraRaju, "Designing and Analysis of 8 Bit SRAM Cell with Low Subthreshold Leakage Power " in International Journal of Modern Engineering Research, Vol.2, Issue.3 (2012).

[11] Eitan N. Shauly," CMOS Leakage and Power Reduction in Transistors and Circuits: Process and Layout Considerations in Journal of low power Electronics(2012).

[12] T. H. Kim, J. Liu, J. Keane, and C. H. Kim, “A 0.2 V, 480 kb sub threshold SRAM with $1 \mathrm{k}$ cells per bit line for ultra-low-voltage computing," IEEE J. Solid-State Circuits, vol. 43, no. 2, (2008) pp. $518-529$.

[13] G. Ramprabu, S. Nagarajan, "Design and Analysis of Novel Modified Cross Layer Controller for WMSN", Indian Journal of Science and Technology, Vol 8(5), March 2015, pp.438-444. 